\title{
ASYMPTOTIC BEHAVIOR OF SOLUTIONS TO THE COMPRESSIBLE NAVIER-STOKES EQUATIONS ON THE HALF SPACE
}

\author{
YOSHIYUKI KAGEI \\ Faculty of Mathematics, Kyushu University \\ Fukuoka 812-8581, Japan \\ E-mail:kagei@math.kyushu-u.ac.jp \\ TAKAYUKI KOBAYASHI \\ Department of Mathematics, Faculty of Science and Engineering \\ Saga University, Saga 840-8502, Japan \\ E-mail: kobayasi@ms.saga-u.ac.jp
}

We consider the initial boundary value problem for the compressible Navier-Stokes equation on the half space $\mathbf{R}_{+}^{n}$ :

$$
\begin{gathered}
\partial_{t} \rho+\operatorname{div} m=0, \\
\partial_{t} m+\operatorname{div}\left(\frac{m \otimes m}{\rho}\right)+\nabla P(\rho)=\nu \Delta\left(\frac{m}{\rho}\right)+(\nu+\tilde{\nu}) \nabla \operatorname{div}\left(\frac{m}{\rho}\right), \\
\left.m\right|_{x_{n}=0}=0, \\
\rho(0, x)=\rho_{0}(x), \quad m(0, x)=m_{0}(x) .
\end{gathered}
$$

Here

$$
\mathbf{R}_{+}^{n}=\left\{x={ }^{T}\left({ }^{T} x^{\prime}, x_{n}\right) ; x^{\prime}={ }^{T}\left(x_{1}, \cdots, x_{n-1}\right) \in \mathbf{R}^{n-1}, x_{n}>0\right\},
$$

$n \geq 2$, and the superscript ${ }^{T}$. stands for the transposition; $\rho=\rho(t, x)$ and $m={ }^{T}\left(m_{1}(t, x)\right.$, $\left.\ldots, m_{n}(t, x)\right)$ denote the unknown density and momentum at time $t \geq 0$ and position $x \in \mathbf{R}_{+}^{n}$, respectively; $P=P(\rho)$ is the pressure; $\nu$ and $\widetilde{\nu}$ are the viscosity coefficients that satisfy $\nu>0, \frac{2}{n} \nu+\widetilde{\nu} \geq 0$; $\operatorname{div} m$ denotes the usual divergence in $x$ of $m$; and $\nabla f$ denotes the usual gradient in $x$ of a scalar function $f$. (e.g., $j$-th component of $\nabla P(\rho)$ is $\partial_{x_{j}} P(\rho)$. The notation $\operatorname{div}\left(\frac{m \otimes m}{\rho}\right)$ means that its $j$-th component is given by $\operatorname{div}\left(\frac{m_{j} m}{\rho}\right)$.

2000 Mathematics Subject Classification: 35Q30, 76N10.

The paper is in final form and no version of it will be published elsewhere. 
In this paper we are interested in the large time behavior of solutions to problem $(0.1)-(0.2)$ around a constant equilibrium ${ }^{T}\left(\rho,{ }^{T} m\right)={ }^{T}\left(\rho^{*}, 0\right)$, where $\rho^{*}$ is a given positive number. We will investigate large time behavior in $L^{p}$ spaces for $2 \leq p \leq \infty$.

We will assume that $P(\rho)$ is smooth and that $\partial_{\rho} P\left(\rho^{*}\right)>0$.

Notations. We denote the $k \times k$ identity matrix by $I_{k}$. In particular, when $k=n+1$, we simply write $I$ for $I_{n+1}$. We also define $(n+1) \times(n+1)$ diagonal matrices $\check{I}$ and $Q_{0}$ by $\check{I}=\operatorname{diag}(1, \cdots, 1,-1)$ and $Q_{0}=\operatorname{diag}(1,0, \cdots, 0)$. Namely,

$$
I=\left(\begin{array}{ccc}
1 & & \\
& \ddots & \\
& & \\
& & 1
\end{array}\right), \quad \check{I}=\left(\begin{array}{cccc}
1 & & & \\
& \ddots & & \\
& & 1 & \\
& & & -1
\end{array}\right), \quad Q_{0}=\left(\begin{array}{cccc}
1 & & & \\
& 0 & & \\
& & \ddots & \\
& & & 0
\end{array}\right) .
$$

For a function $f=f\left(x^{\prime}\right)\left(x^{\prime} \in \mathbf{R}^{n-1}\right)$, we denote its Fourier transform by $\mathscr{F}_{x^{\prime}} f$ :

$$
\left(\mathscr{F}_{x^{\prime}} f\right)\left(\xi^{\prime}\right)=\int_{\mathbf{R}^{n-1}} f\left(x^{\prime}\right) e^{-i x^{\prime} \cdot \xi^{\prime}} d x^{\prime}
$$

We also define $q(\ell)$ for $\ell \geq 0$ by

$$
q(\ell)= \begin{cases}\min \left\{\frac{\ell}{2}, \frac{\ell}{4}+\frac{1}{8}\right\} & (n=2) \\ \min \left\{\frac{\ell}{2}, \frac{\ell}{4}+\frac{3}{8}\right\} & (n \geq 3)\end{cases}
$$

and $k_{p}(\ell)$ for $\ell \in \mathbf{R}$ and $2 \leq p \leq \infty$ by

$$
k_{p}(\ell)= \begin{cases}\max \left\{0, \ell+n\left(\frac{1}{2}-\frac{1}{p}\right)\right\} & \text { when } p=2, \\ \max \left\{0,\left[\ell+n\left(\frac{1}{2}-\frac{1}{p}\right)\right]\right\}+1 & \text { when } 2<p \leq \infty,\end{cases}
$$

where $[q]$ denotes the greatest integer less than or equal to $q$.

1. Linearized problem. The linearized problem takes the form, after a suitable change of variables,

$$
\begin{aligned}
\partial_{t} \rho+\gamma \operatorname{div} m & =0, \\
\partial_{t} m-\alpha \Delta m-\beta \nabla \operatorname{div} m+\gamma \nabla \rho & =0, \\
\left.m\right|_{x_{n}}=0 ;\left.\quad\left(\begin{array}{c}
\rho \\
m
\end{array}\right)\right|_{t=0}=\left(\begin{array}{c}
\rho_{0} \\
m_{0}
\end{array}\right) & \equiv u_{0},
\end{aligned}
$$

where $\alpha, \beta$ and $\gamma$ are positive constants. Hereafter we denote the solution $\left(\begin{array}{c}\rho(t) \\ m(t)\end{array}\right)$ of problem (1.1)-(1.2) by $\mathscr{U}(t) u_{0}$ :

$$
\mathscr{U}(t) u_{0}=\left(\begin{array}{c}
\rho(t) \\
m(t)
\end{array}\right)
$$

To understand the behavior of the solution to problem (1.1)-(1.2), we briefly observe the Cauchy problem for (1.1) on the whole space $\mathbf{R}^{n}$. Let us denote by $\mathscr{E}(t, x)$ the fundamental solution of the Cauchy problem. Then the Fourier transform of $\mathscr{E}(t, x)$, which 
is denoted by $\mathscr{A}(t, \xi)\left(\xi \in \mathbf{R}^{n}\right)$, is given by

$$
\begin{aligned}
\mathscr{A}(t, \xi)= & e^{-\alpha|\xi|^{2} t}\left(\begin{array}{cc}
0 & 0 \\
0 x & I_{n}-\frac{\xi^{T} \xi}{|\xi|^{2}}
\end{array}\right) \\
& +\left\{\frac{e^{\lambda_{+} t}}{\lambda_{+}-\lambda_{-}}\left(\begin{array}{cc}
-\lambda_{-} & -i \gamma^{T} \xi \\
-i \gamma \xi & \lambda_{+} \frac{\xi^{T} \xi}{|\xi|^{2}}
\end{array}\right)+\frac{e^{\lambda_{-} t}}{\lambda_{-}-\lambda_{+}}\left(\begin{array}{cc}
-\lambda_{+} & -i \gamma^{T} \xi \\
-i \gamma \xi & \lambda_{-} \frac{\xi^{T} \xi}{|\xi|^{2}}
\end{array}\right)\right\},
\end{aligned}
$$

Here

$$
\lambda_{ \pm}=-a_{1}|\xi|^{2} \pm \frac{1}{2} \sqrt{(\alpha+\beta)^{2}|\xi|^{4}-4 \gamma^{2}|\xi|^{2}}, \quad a_{1}=\frac{1}{2}(\alpha+\beta) .
$$

Since

$$
\lambda_{ \pm} \sim-a_{1}|\xi|^{2} \pm i \gamma|\xi| \quad \text { for } \quad|\xi|<<1
$$

and

$$
\lambda_{+} \sim-a_{0}, \quad \lambda_{-} \sim-2 a_{1}|\xi|^{2} \text { for }|\xi|>>1,
$$

where $a_{0}=\frac{\gamma^{2}}{\alpha+\beta}$, we see that

$$
\mathscr{A} \sim e^{-\alpha|\xi|^{2} t} \widehat{E}_{D}(\xi)+\frac{1}{2} e^{-a_{1}|\xi|^{2} t}\left\{e^{i \gamma|\xi| t} \widehat{E}_{D W,+}(\xi)+e^{-i \gamma|\xi| t} \widehat{E}_{D W,-}(\xi)\right\}
$$

for $|\xi|<<1$, and

$$
\mathscr{A} \sim e^{-a_{0} t} \widehat{E}_{\infty}(\xi)
$$

for $|\xi|>>1$, where

$$
\widehat{E}_{D}(\xi)=\left(\begin{array}{cc}
0 & 0 \\
0 & I_{n}-\frac{\xi^{T} \xi}{|\xi|^{2}}
\end{array}\right), \quad \widehat{E}_{D W, \pm}(\xi)=\left(\begin{array}{cc}
1 & \mp \frac{{ }^{T} \xi}{|\xi|} \\
\mp \frac{\xi}{|\xi|} & \frac{\xi^{T} \xi}{|\xi|^{2}}
\end{array}\right)
$$

and

$$
\widehat{E}_{\infty}(\xi)=\left(\begin{array}{cc}
1 & \frac{i \gamma}{2 a_{1}} \frac{T^{T} \xi}{|\xi|^{2}} \\
\frac{i \gamma}{2 a_{1}} \frac{\xi}{|\xi|^{2}} & -\frac{a_{0}}{2 a_{1}} \frac{\xi^{T} \xi}{|\xi|^{4}}
\end{array}\right) .
$$

Therefore, the asymptotically leading part is given by the low frequency part $|\xi|<<1$. The first term for $|\xi|<<1$ gives the heat kernel. The second term for $|\xi|<<1$ is the Gaussian with oscillatory factor, and, thus, yields the convolution of the heat kernel and the fundamental solution of the wave equation, which is the so-called diffusion wave.

It then turns out that the solution $\mathscr{E}(t, \cdot) * u_{0}$ of the Cauchy problem is decomposed as

$$
\mathscr{E}(t, \cdot) * u_{0}=\mathscr{E}_{D}(t, \cdot) * u_{0}+\mathscr{E}_{D W}(t, \cdot) * u_{0}+\mathscr{E}_{\infty}(t, \cdot) * u_{0}
$$

where $*$ denotes the convolution in $x \in \mathbf{R}^{n}$,

$$
\begin{gathered}
\mathscr{E}_{D}(t, x) \sim \mathscr{F}_{x}^{1}\left[e^{-\alpha|\xi|^{2} t} \widehat{E}_{D}(\xi)\right], \\
\mathscr{E}_{D W}(t, x) \sim \mathscr{F}_{x}{ }^{1}\left[\frac{1}{2} e^{-a_{1}|\xi|^{2} t}\left\{e^{i \gamma|\xi| t} \widehat{E}_{D W,+}(\xi)+e^{-i \gamma|\xi| t} \widehat{E}_{D W,-}(\xi)\right\}\right], \\
\mathscr{E}_{\infty}(t, x) \sim e^{-a_{0} t} \mathscr{F}_{x}{ }^{1}\left[\widehat{E}_{\infty}(\xi)\right],
\end{gathered}
$$

and each term is estimated as follows for $\ell \neq 0$ :

$$
\left\|\partial_{x}^{\ell} \mathscr{E}_{D}(t, \cdot) * u_{0}\right\|_{L^{p}\left(\mathbf{R}^{n}\right)} \leq C t^{-\frac{n}{2}\left(1-\frac{1}{p}\right)-\frac{|\ell|}{2}}\left\|u_{0}\right\|_{L^{1}\left(\mathbf{R}^{n}\right)},
$$




$$
\begin{aligned}
\left\|\partial_{x}^{\ell} \mathscr{E}_{D W}(t, \cdot) * u_{0}\right\|_{L^{p}\left(\mathbf{R}^{n}\right)} & \leq C t^{-\frac{n}{2}\left(1-\frac{1}{p}\right)-\frac{n-1}{4}\left(1-\frac{2}{p}\right)-\frac{|\ell|}{2}}\left\|u_{0}\right\|_{L^{1}\left(\mathbf{R}^{n}\right)}, \\
\left\|\partial_{x}^{\ell} \mathscr{E}_{\infty}(t, \cdot) * u_{0}\right\|_{L^{p}\left(\mathbf{R}^{n}\right)} & \leq C e^{-c_{0} t}\left(\left\|m_{0}\right\|_{H^{k_{p}(|\ell|-1)}\left(\mathbf{R}^{n}\right)}+\left\|\rho_{0}\right\|_{H^{k(|\ell|)}\left(\mathbf{R}^{n}\right)}\right) .
\end{aligned}
$$

Here, note that the factor $t^{-\frac{n-1}{4}\left(1-\frac{2}{p}\right)}$ in the estimate for the diffusion wave $\mathscr{E}_{D W}(t, \cdot) * u_{0}$ arises from the spreading effect of the oscillatory factor $e^{ \pm i \gamma|\xi| t}$. Also when $\ell=0$, the similar results hold. See $[2,10]$ for more details.

Let us now consider the half space problem (1.1)-(1.2). For this problem, as we will see below, the solution $\mathscr{U}(t) u_{0}$ is written in the sum of $\mathscr{U}^{(C)}(t) u_{0}$ and $\mathscr{U}^{(H)}(t) u_{0}$, where $\mathscr{U}^{(C)}(t) u_{0}$ can be obtained by a simple reflection argument from the solution of the Cauchy problem, while $\mathscr{U}^{(H)}(t) u_{0}$ cannot be obtained by reflection argument from the Cauchy problem. We will see that the asymptotically leading part of $\mathscr{U}(t) u_{0}$ is given by the sum of two terms, similarly to the Cauchy problem, one of which behaves like purely diffusively and the other one behaves like diffusion waves. However, there appear some aspects different from the Cauchy problem, due to $\mathscr{U}^{(H)}(t) u_{0}$.

TheOREM 1.1. Let $\mathscr{U}(t) u_{0}$ be the solution of problem (1.1)-(1.2). Then $\mathscr{U}(t) u_{0}$ is decomposed as

$$
\mathscr{U}(t) u_{0}=\mathscr{U}_{D}(t) u_{0}+\mathscr{U}_{D W}(t) u_{0}+\mathscr{U}_{\infty}(t) u_{0},
$$

where $\mathscr{U}_{D}(t) u_{0}$ and $\mathscr{U}_{D W}(t) u_{0}$ take the form in (i) and (ii), respectively, and each term on the right-hand side has the estimates in (iii).

(i) $\mathscr{U}_{D}(t) u_{0}$ behaves like purely diffusively and it is written in the form

$$
\begin{aligned}
& \mathscr{U}_{D}(t) u_{0}=\mathscr{F}_{x^{\prime}}^{1}\left[\widehat{\mathscr{U}}_{D}(t) \widehat{u}_{0}\right], \\
& \widehat{\mathscr{U}}_{D}(t) \widehat{u}_{0}=\int_{0}^{\infty} \widehat{U}_{D}\left(t, \xi^{\prime}, x_{n}, y_{n}\right) \widehat{u}_{0}\left(\xi^{\prime}, y_{n}\right) d y_{n}
\end{aligned}
$$

with

$$
\widehat{U}_{D}\left(t, \xi^{\prime}, x_{n}, y_{n}\right)=\frac{1}{2 \pi} \int_{\mathbf{R}} e^{-\alpha|\xi|^{2} t} \widehat{L}_{D}\left(\xi^{\prime}, \xi_{n}, x_{n}, y_{n}\right) d \xi_{n}
$$

for some function $\widehat{L}_{D}\left(\xi^{\prime}, \xi_{n}, x_{n}, y_{n}\right)$.

(ii) $\mathscr{U}_{D W}(t) u_{0}$ behaves like diffusion waves and it is written in the form

$$
\mathscr{U}_{D W}(t) u_{0} \sim \mathscr{U}_{D W}^{(0)}(t) u_{0} \quad(t \rightarrow \infty)
$$

where

$$
\begin{aligned}
& \mathscr{U}_{D W}^{(0)}(t) u_{0}=\mathscr{F}_{x^{\prime}}^{1}\left[\widehat{\mathscr{U}}_{D W}^{(0)}(t) \widehat{u}_{0}\right] \\
& \widehat{\mathscr{U}}_{D W}^{(0)}(t) \widehat{u}_{0}=\int_{0}^{\infty} \widehat{U}_{D W}^{(0)}\left(t, \xi^{\prime}, x_{n}, y_{n}\right) \widehat{u}_{0}\left(\xi^{\prime}, y_{n}\right) d y_{n}
\end{aligned}
$$

and

$$
\begin{aligned}
& \widehat{U}_{D W}^{(0)}\left(t, \xi^{\prime}, x_{n}, y_{n}\right) \\
& =\frac{1}{2 \pi} \int_{\mathbf{R}} e^{-a_{1}|\xi|^{2} t}\left\{e^{i \gamma|\xi| t} \widehat{G}_{D W,+}\left(\xi^{\prime}, \xi_{n}, x_{n}, y_{n}\right)+e^{-i \gamma|\xi| t} \widehat{G}_{D W,-}\left(\xi^{\prime}, \xi_{n}, x_{n}, y_{n}\right)\right\} d \xi_{n} \\
& \quad+e^{-\widetilde{a}_{1}\left|\xi^{\prime}\right|^{2} t}\left\{e^{i \gamma\left|\xi^{\prime}\right| t} \widehat{G}_{D W,+}^{(R e s)}\left(\xi^{\prime}, x_{n}, y_{n}\right)+e^{-i \gamma\left|\xi^{\prime}\right| t} \widehat{G}_{D W,-}^{(R e s)}\left(\xi^{\prime}, x_{n}, y_{n}\right)\right\}
\end{aligned}
$$


Here $a_{1}=\frac{1}{2}(\alpha+\beta), \widetilde{a}_{1}=\frac{1}{2}(2 \alpha+\beta)$,

$$
\begin{aligned}
\widehat{G}_{D W, \pm}\left(\xi^{\prime}, \xi_{n}, x_{n}, y_{n}\right)= & \frac{1}{2} \widehat{E}_{D W, \pm}\left(\xi^{\prime}, \xi_{n}\right)\left\{e^{i \xi_{n}\left(x_{n}-y_{n}\right)} I+e^{i \xi_{n}\left(x_{n}+y_{n}\right)} \check{I}\right\} \\
+ & \widehat{G}_{D W, \pm}^{(1)}\left(\xi^{\prime}, \xi_{n}\right) e^{i \xi_{n} x_{n}} e^{-a_{2}^{( \pm)}|\xi|^{1 / 2} y_{n}}\left(I-Q_{0}\right) \\
+ & \left\{\widehat{G}_{D W, \pm}^{(2)}\left(\xi^{\prime}, \xi_{n}\right) e^{i \xi_{n} x_{n}}+\widehat{G}_{D W, \pm}^{(3)}\left(\xi^{\prime}, \xi_{n}\right) e^{-a_{2}^{( \pm)}|\xi|^{1 / 2} x_{n}}\right\} \\
& \times\left\{e^{i \xi_{n} y_{n}} Q_{0}+\left(e^{-a_{2}^{( \pm)}|\xi|^{1 / 2} y_{n}}-e^{i \xi_{n} y_{n}}\right)\left(I-Q_{0}\right)\right\}
\end{aligned}
$$

with $\xi={ }^{T}\left({ }^{T} \xi^{\prime}, \xi_{n}\right), a_{2}^{( \pm)}=e^{ \pm i \frac{\pi}{4}} \sqrt{\frac{\gamma}{\alpha}}, \widehat{E}_{D W, \pm}\left(\xi, \xi_{n}\right)$ being defined in (1.3), and $I, \check{I}$ and $Q_{0}$ being defined (0.3), and

$$
\begin{gathered}
\widehat{G}_{D W, \pm}^{(1)}\left(\xi^{\prime}, \xi_{n}\right)=\left(\begin{array}{ccc}
0 & \pm \frac{{ }^{T} \xi^{\prime}}{|\xi|} & 0 \\
0 & -\frac{\xi^{\prime T} \xi^{\prime}}{|\xi|^{2}} & 0 \\
0 & -\frac{\xi_{n}^{T} \xi^{\prime}}{|\xi|^{2}} & 0
\end{array}\right), \\
\widehat{G}_{D W, \pm}^{(2)}\left(\xi^{\prime}, \xi_{n}\right)=-\frac{\left|\xi^{\prime}\right|^{2}}{i a_{2}^{( \pm)}|\xi|^{1 / 2} \xi_{n}+\left|\xi^{\prime}\right|^{2}}\left(\begin{array}{ccc}
1 & \pm \frac{\xi^{\prime}}{|\xi|} & \mp \frac{\xi_{n}}{|\xi|} \\
\mp \frac{\xi^{\prime}}{|\xi|} & -\frac{\xi^{\prime T} \xi^{\prime}}{|\xi|^{2}} & \frac{\xi^{\prime} \xi_{n}}{|\xi|^{2}} \\
\mp \frac{\xi_{n}}{|\xi|} & -\frac{\xi_{n} \xi^{\prime}}{|\xi|^{2}} & \frac{\xi_{n}^{2}}{|\xi|^{2}}
\end{array}\right), \\
\widehat{G}_{D W, \pm}^{(3)}\left(\xi^{\prime}, \xi_{n}\right)=\frac{\gamma}{a_{2}^{( \pm)} \alpha} \frac{\xi_{n}}{|\xi|^{1 / 2}} \frac{}{i a_{2}^{( \pm)}|\xi|^{1 / 2} \xi_{n}+\left|\xi^{\prime}\right|^{2}}\left(\begin{array}{ccc}
0 & 0 & 0 \\
-\xi^{\prime} & \mp \frac{\xi^{\prime T} \xi^{\prime}}{|\xi|} & \pm \frac{\xi^{\prime} \xi_{n}}{|\xi|} \\
0 & 0 & 0
\end{array}\right) ;
\end{gathered}
$$

and

$$
\begin{aligned}
\widehat{G}_{D W, \pm}^{(R e s)}\left(\xi^{\prime}, x_{n}, y_{n}\right)= & \left\{\widehat{G}_{D W, \pm}^{(R e s, 1)}\left(\xi^{\prime}\right) e^{-a_{2}^{( \pm)}\left|\xi^{\prime}\right|^{1 / 2} x_{n}}+\widehat{G}_{D W, \pm}^{(R e s, 2)}\left(\xi^{\prime}\right) e^{-\frac{1}{a_{2}^{( \pm)}}\left|\xi^{\prime}\right|^{3 / 2} x_{n}}\right\} \\
& \times\left\{e^{-\frac{1}{a_{2}^{( \pm)}}\left|\xi^{\prime}\right|^{3 / 2} y_{n}} Q_{0}+\left(e^{-a_{2}^{( \pm)}\left|\xi^{\prime}\right|^{1 / 2} y_{n}}-e^{-\frac{1}{a_{2}^{( \pm)}}\left|\xi^{\prime}\right|^{3 / 2} y_{n}}\right)\left(I-Q_{0}\right)\right\}
\end{aligned}
$$

with

$$
\begin{gathered}
\widehat{G}_{D W, \pm}^{(R e s, 1)}\left(\xi^{\prime}\right)=\frac{1}{a_{2}^{( \pm)}}\left(\begin{array}{ccc}
0 & 0 & 0 \\
\mp \xi^{\prime}\left|\xi^{\prime}\right|^{1 / 2} & -\frac{\xi^{\prime T} \xi^{\prime}}{\left|\xi^{\prime}\right|^{1 / 2}} & 0 \\
0 & 0 & 0
\end{array}\right), \\
\widehat{G}_{D W, \pm}^{(R e s, 2)}\left(\xi^{\prime}\right)=i \frac{a_{2}^{( \pm)} \alpha}{\gamma}\left(\begin{array}{ccc}
\mp\left|\xi^{\prime}\right|^{3 / 2} & -\left|\xi^{\prime}\right|^{1 / 2 T} \xi^{\prime} & 0 \\
\xi^{\prime}\left|\xi^{\prime}\right|^{3 / 2} & \pm \frac{\xi^{T} \xi^{\prime}}{\left|\xi^{\prime}\right|^{1 / 2}} & 0 \\
0 & 0 & 0
\end{array}\right) .
\end{gathered}
$$

(iii) Let $\ell$ be a multi-index and let $2 \leq p \leq \infty$. Then there exist constants $c_{0}>0$ and $C>0$ such that

$$
\begin{aligned}
\left\|\partial_{x}^{\ell} \mathscr{U}_{D}(t) u_{0}\right\|_{p} & \leq C t^{-\frac{n}{2}\left(1-\frac{1}{p}\right)-\frac{|\ell|}{2}}\left\|u_{0}\right\|_{1}, \\
\left\|\partial_{x}^{\ell} \mathscr{U}_{D W}(t) u_{0}\right\|_{p} & \leq C t^{-\frac{n}{2}\left(1-\frac{1}{p}\right)-\frac{n-1}{4}\left(1-\frac{2}{p}\right)-\frac{2}{p} q(|\ell|)-\left(1-\frac{2}{p}\right) \frac{|\ell|}{4}}\left\|u_{0}\right\|_{1}, \\
\left\|\partial_{x}^{\ell} \mathscr{U}_{\infty}(t) u_{0}\right\|_{p} & \leq C e^{-c_{0} t}\left(\left\|m_{0}\right\|_{H^{k_{p}(|\ell|-1)}}+\left\|\rho_{0}\right\|_{\left.H^{k_{p}(|\ell|)}\right) .}\right.
\end{aligned}
$$

for all $t \geq 1$. Here $q(|\ell|)$ and $k_{p}(|\ell|)$ are defined in (0.4) and (0.5), respectively. 
Corollary 1.2. Assume that $n \geq 2$. Let $\ell$ be a multi-index and let $2 \leq p \leq \infty$. Then, there exist positive constants $c_{0}$ and $C$ such that the following estimates in (i) and (ii) hold for all $t \geq 1$.

(i) If $|\ell| \leq n-1$, then $\left\|\partial_{x}^{\ell} \mathscr{U}(t) u_{0}\right\|_{p} \leq C\left\{t^{-\frac{n}{2}\left(1-\frac{1}{p}\right)-\frac{2}{p} q(|\ell|)-\left(1-\frac{2}{p}\right) \frac{|\ell|}{2}}\left\|u_{0}\right\|_{1}+e^{-c_{0} t}\left(\left\|m_{0}\right\|_{H^{k_{p}(|\ell|-1)}}+\left\|\rho_{0}\right\|_{\left.H^{k_{p}(|\ell|)}\right)}\right)\right.$.

(ii) If $|\ell| \geq n$, then

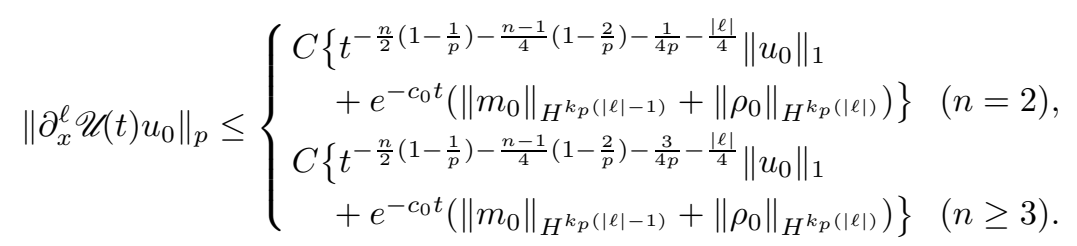

REMARK. The diffusive part $\mathscr{U}_{D}(t) u_{0}$ is approximated by the incompressible flow in large time, i.e.,

$$
\mathscr{U}_{D}(t) u_{0} \sim\left(\begin{array}{c}
\tilde{p}(t) \\
\widetilde{v}(t)
\end{array}\right) \text { as } t \rightarrow \infty,
$$

where $\left(\begin{array}{c}\widetilde{p}(t) \\ \widetilde{v}(t)\end{array}\right)$ is the solution of the nonstationary Stokes problem

$$
\begin{aligned}
\operatorname{div} \widetilde{v} & =0, \\
\partial_{t} \widetilde{v}-\alpha \Delta \widetilde{v}+\gamma \nabla \widetilde{p} & =0, \\
\left.\widetilde{v}\right|_{x_{n}=0}=0,\left.\quad \widetilde{v}\right|_{t=0} & =\mathbb{P} m_{0}
\end{aligned}
$$

with $\widetilde{p}(t) \in L^{\widetilde{r}}\left(\mathbf{R}_{+}^{n}\right)$ for all $t>0$ and some fixed $2<\widetilde{r}<\infty$. Here $\mathbb{P}$ denotes the orthogonal projector from $\left\{L^{2}\left(\mathbf{R}_{+}^{n}\right)\right\}^{n}$ onto the solenoidal subspace associated with the Helmholtz decomposition of $\left\{L^{2}\left(\mathbf{R}_{+}^{n}\right)\right\}^{n}$.

To analyze the nonlinear problem (0.1)-(0.2), we will make use of estimate for $\mathscr{U}_{D}(t)\left[\partial_{x} u_{0}\right]:$

TheOREM 1.5. Let $u_{0}=\left(\begin{array}{c}0 \\ m_{0}\end{array}\right)$ and let $2 \leq p \leq \infty$. Then there exist constants $c_{0}>0$ and $C>0$ such that

$$
\begin{aligned}
\left\|\mathscr{U}_{D}(t)\left[\partial_{x} u_{0}\right]\right\|_{p} & \leq C t^{-\frac{n}{2}\left(1-\frac{1}{p}\right)-\frac{1}{2}}\left\|m_{0}\right\|_{1}, \\
\left\|\mathscr{U}_{D W}(t)\left[\partial_{x} u_{0}\right]\right\|_{p} & \leq C t^{-\frac{n}{2}\left(1-\frac{1}{p}\right)-\frac{n-1}{4}\left(1-\frac{2}{p}\right)-\frac{1}{4}}\left\|m_{0}\right\|_{1}, \\
\left\|\mathscr{U}_{\infty}(t)\left[\partial_{x} u_{0}\right]\right\|_{p} & \leq C e^{-c_{0} t}\left\|m_{0}\right\|_{H^{k_{p}}(0)} .
\end{aligned}
$$

for all $t \geq 1$. As a result,

$$
\left\|\mathscr{U}(t)\left[\partial_{x} u_{0}\right]\right\|_{p} \leq C t^{-\frac{n}{2}\left(1-\frac{1}{p}\right)-\frac{1}{2}\left(1-\frac{1}{p}\right)}\left(\left\|m_{0}\right\|_{1}+\left\|m_{0}\right\|_{H^{k}(0)}\right) .
$$

Remarks. (i) In the case of the Cauchy problem, $\left\|\mathscr{E}(t)\left[\partial_{x} u_{0}\right]\right\|_{L^{p}\left(\mathbf{R}^{n}\right)}=O\left(t^{-\frac{n}{2}\left(1-\frac{1}{p}\right)-\frac{1}{2}}\right)$.

(ii) The estimate $\left\|\mathscr{U}(t)\left[\partial_{x} u_{0}\right]\right\|_{2}=O\left(t^{-\frac{n}{4}-\frac{1}{4}}\right)$ is optimal for all $n \geq 2$.

(iii) The slower decay rate arises from the terms with $e^{-a_{2}^{( \pm)}|\xi|^{1 / 2} y_{n}}$ in the representation of $\mathscr{U}_{D W}(t)\left[\partial_{x} u_{0}\right]$. In particular, the term with 


$$
e^{-a_{1}|\xi|^{2} t \pm i \gamma|\xi| t} \widehat{G}_{D W, \pm}^{(1)}\left(\xi^{\prime}, \xi_{n}\right) e^{i \xi_{n} x_{n}}\left(-a_{2}^{( \pm)}|\xi|^{1 / 2}\right) e^{-a_{2}^{( \pm)}|\xi|^{1 / 2} y_{n}}\left(I-Q_{0}\right)
$$

decays in $L^{2}$ norm as $O\left(t^{-\frac{n}{4}-\frac{1}{4}}\right)$.

2. Nonlinear problem. We next present our results on the large time behavior of solutions to the nonlinear problem $(0.1)-(0.2)$. In what follows we write $s_{0}$ for $\left[\frac{n}{2}\right]+1$. We first state the assumptions on the data.

Assumptions.

(A1) The pressure $P=P(\rho)$ is a smooth function of $\rho$ in a neighborhood of $\rho^{*}$ and $\frac{\partial P}{\partial \rho}\left(\rho^{*}\right)>0$.

(A2) The initial perturbation ${ }^{T}\left(\rho_{0}-\rho^{*},{ }^{T} m_{0}\right)$ belongs to $H^{s}$ for some $s \in \mathbf{Z}$ with $s \geq s_{0}+1$ and satisfies a suitable compatibility condition.

TheOrem 2.1. (i) Let $n \geq 2$. Assume that (A1) and (A2) hold for some $s \in \mathbf{Z}$ with $s \geq s_{0}+1$. Then there exists a positive number $\eta_{0}$ such that if the initial perturbation ${ }^{T}\left(\rho_{0}-\rho^{*},{ }^{T} m_{0}\right) \in H^{s} \cap L^{1}$ and if $\left\|^{T}\left(\rho_{0}-\rho^{*},{ }^{T} m_{0}\right)\right\|_{H^{s}}+\left\|^{T}\left(\rho_{0}-\rho^{*},{ }^{T} m_{0}\right)\right\|_{L^{1}} \leq \eta_{0}$, then the perturbation $u(t) \equiv{ }^{T}\left(\rho(t)-\rho^{*},{ }^{T} m(t)\right)$ satisfies

$$
\|u(t)\|_{p}=O\left(t^{-\frac{n}{2}\left(1-\frac{1}{p}\right)}\right)
$$

and

$$
\left\|\partial_{x} u(t)\right\|_{2}= \begin{cases}O\left(t^{-\frac{n}{4}-\frac{3}{8}}\right) & (n=2) \\ O\left(t^{-\frac{n}{4}-\frac{1}{2}}\right) & (n \geq 3)\end{cases}
$$

for $2 \leq p \leq \infty$ as $t \rightarrow \infty$.

(ii) For $u_{0}={ }^{T}\left(\bar{\rho}_{0},{ }^{T} \bar{m}_{0}\right)$ with $\bar{\rho}_{0} \in H^{1}$ and $\bar{m}_{0}={ }^{T}\left(\bar{m}_{0,1}, \cdots, \bar{m}_{0, n}\right) \in L^{2}$, let $\mathscr{U}(t) u_{0}(x)=$ ${ }^{T}\left(\bar{\rho}(t, x),{ }^{T} \bar{m}(t, x)\right)$ denote the solution of the linearized problem at ${ }^{T}\left(\rho^{*}, 0\right)$. Then, under the same assumptions on ${ }^{T}\left(\rho_{0}-\rho^{*},{ }^{T} m_{0}\right)$ in $(\mathrm{i})$,

$$
\left\|u(t)-\mathscr{U}(t) u_{0}\right\|_{2}=O\left(t^{-\frac{n}{4}-\frac{1}{4}} L(t)\right)
$$

as $t \rightarrow \infty$, where $u_{0}={ }^{T}\left(\rho_{0}-\rho^{*},{ }^{T} m_{0}\right)$, and $L(t)=\log (1+t)$ when $n=2$ and $L(t)=1$ for $n \geq 3$.

(iii) Assume, in addition, that (A2) holds for some $s \in \mathbf{Z}$ with $s \geq s_{0}+2$. Then there exists a positive number $\tilde{\eta}_{0}$ such that If, $\left\|^{T}\left(\rho_{0}-\rho^{*},{ }^{T} m_{0}\right)\right\|_{H^{s}}+\left\|^{T}\left(\rho_{0}-\rho^{*},{ }^{T} m_{0}\right)\right\|_{L_{1}} \leq \tilde{\eta}_{0}$,

$$
\left\|u(t)-\mathscr{U}(t) u_{0}\right\|_{\infty}= \begin{cases}O\left(t^{-\frac{n}{2}-\frac{3}{8}} \log t\right) & (n=2), \\ O\left(t^{-\frac{n}{2}-\frac{1}{2}}\right) & (n \geq 3),\end{cases}
$$

as $t \rightarrow \infty$, where $u_{0}={ }^{T}\left(\rho_{0}-\rho^{*},{ }^{T} m_{0}\right)$.

REMARKs. (i) Since $\left\|\mathscr{U}(t) u_{0}\right\|_{p}=O\left(t^{-\frac{n}{2}\left(1-\frac{1}{p}\right)}\right)$, the estimates in (ii) and (iii) mean that the leading part of $u(t)$ in large time is given by $\mathscr{U}(t) u_{0}$, similarly to the case of the Cauchy problem $[2,6,7]$.

(ii) The estimates in (ii) and (iii) are obtained via Duhamel's principle

$$
\left.u(t)=\mathscr{U}(t) u_{0}+\int_{0}^{t} \mathscr{U}(t-s)\left[\operatorname{div} \mathscr{N} u, \partial_{x} u\right)(s)\right] d s,
$$

by applying the estimate for $\mathscr{U}(t)\left[\partial_{x} u_{0}\right]$ to $\mathscr{U}(t-s)\left[\operatorname{div} \mathscr{N}\left(u, \partial_{x} u\right)(s)\right]$. Here $\operatorname{div} \mathscr{M}\left(u, \partial_{x} u\right)$ 
denotes the nonlinearity. From this identity we may say that the leading part of $u(t)-$ $\mathscr{U}(t) u_{0}$ is given by the nonlinear interaction of the leading part of $\mathscr{U}(t) u_{0}$. The decay rate in (ii) is slower than the corresponding one for the Cauchy problem, but, the estimate $\left\|\mathscr{U}(t)\left[\partial_{x} u_{0}\right]\right\|_{2}=O\left(t^{-\frac{n}{4}-\frac{1}{4}}\right)$ is optimal. This would, therefore, indicate that the leading part of the nonlinear interaction in $L^{2}$ space may involve some slowly decaying terms not appearing in the Cauchy problem.

\section{References}

[1] K. Deckelnick, Decay estimates for the compressible Navier-Stokes equations in unbounded domain, Math. Z. 209 (1992), 115-130.

[2] D. Hoff and K. Zumbrun, Multi-dimensional diffusion waves for the Navier-Stokes equations of compressible flow, Indiana Univ. Math. J. 44 (1995), 604-676.

[3] D. Hoff and K. Zumbrun, Pointwise decay estimates for multidimensional Navier-Stokes diffusion waves, Z. Angew. Math. Phys. 48 (1997), 597-614.

[4] Y. Kagei and T. Kobayashi, On large time behavior of solutions to the Compressible Navier-Stokes Equations in the half space in $R^{3}$, Arch. Rational Mech. Anal. 165 (2002), 89-159.

[5] Y. Kagei and T. Kobayashi, Asymptotic behavior of solutions to the compressible NavierStokes equations on the half space, in preparation.

[6] S. Kawashima, Systems of a hyperbolic-parabolic composite type, with applications to the equations of magnethydrodynamics, Ph. D. Thesis, Kyoto University, 1983.

[7] S. Kawashima, A. Matsumura and T. Nishida, On the fluid-dynamical approximation to the Boltzmann equation at the level of the Navier-Stokes equation, Comm. Math. Phys. 70 (1979), 97-124.

[8] T. Kobayashi, Some estimates of solutions for the equations of motion of compressible viscous fluid in an exterior domain in $R^{3}$, J. Differential Equations 184 (2002), 587-619.

[9] T. Kobayashi and Y. Shibata, Decay estimates of solutions for the equations of motion of compressible viscous and heat-conductive gases in an exterior domain in $R^{3}$, Comm. Math. Phys. 200 (1999), 621-659.

[10] T. Kobayashi and Y. Shibata., Remarks on the rate of decay of solutions to linearized compressible Navier-Stokes equations, Pacific J. Math. 207 (2002), 199-234.

[11] T.-P. Liu and W. Wang, The pointwise estimates of diffusion wave for the Navier-Stokes Systems in odd multi-dimensions, Comm. Math. Phys. 196 (1998), 145-173.

[12] A. Matsumura, An energy method for the equations of motion of compressible viscous and heat-conductive fluids, University of Wisconsin-Madison, MRC Technical Summary Report \# 2194 (1981), 1-16.

[13] A. Matsumura and T. Nishida, The initial value problem for the equations of motion of compressible viscous and heat-conductive fluids, Proc. Japan Acad. Ser. A 55 (1979), $337-342$.

[14] A. Matsumura and T. Nishida, The initial value problems for the equations of motion of viscous and heat-conductive gases, J. Math. Kyoto Univ. 20 (1980), pp.67-104.

[15] A. Matsumura and T. Nishida, Initial boundary value problems for the equations of motion of compressible viscous and heat-conductive fluids, Comm. Math. Phys. 89 (1983), 445-464.

[16] G. Ponce, Global existence of small solutions to a class of nonlinear evolution equations, Nonlinear Anal. 9 (1985), 399-418. 